Journal of

HIV/AIDS \& Infectious Diseases

\title{
Factors Associated with Knowledge of Transmission and Prevention of HIV/ AIDS among Men and Women in Uganda: Evidence from Demographic Health Survey of Uganda 2016
}

\author{
Twinomugisha Fred ${ }^{1, *}$, Suresh Jungari ${ }^{2}$, Govind Chauhan ${ }^{3}$, Muwanguzi Denis ${ }^{4}$ \\ ${ }^{1}$ Chemistry Kyambogo University, Uganda, Master of public health (MPH) Interdisciplinary School of Health Sciences, \\ Savitribai Phule Pune University, Pune, India \\ ${ }^{2}$ Assistant Professor, Interdisciplinary School of Health Sciences, Savitribai Phule Pune University, Pune, India \\ ${ }^{3}$ Assistant professor, Gokhale institute of politics and economics, Pune, India \\ ${ }^{4}$ Director of programs-Budondo inter cultural center ( Bi-centre), Co-founder, Suubi Health Center, Master of public \\ health (MPH) Lund University, Sweden
}

${ }^{\star}$ Corresponding author: Twinomugisha Fred, Chemistry Kyambogo University, Uganda, Master of public health (MPH) Interdisciplinary School of Health Sciences, Savitribai Phule Pune University, Pune, India; Email: twinomugishafred@gmail.com Received Date: July 03, 2019 Accepted Date: August 13, 2019 Published Date: August 16, 2019

Citation: Twinomugisha Fred (2019) Factors Associated With Knowledge of Transmission and Prevention of HIV/AIDS among Men and Women in Uganda: Evidence from Demographic Health Survey of Uganda. J HIV AIDS Infect Dis 5: 1-13.

\section{Back ground}

HIV/AIDS has become a major public health concern globally with majority of those affected found in Sub-Saharan Africa. Knowledge regarding transmission and prevention of HIV/AIDS in Uganda is still low making most persons not to disclose their HIV status due to fear of public judgement and isolation and this makes prevention and treatment very difficult.

Aim: This study aims to examine factors associated with knowledge of HIV/AIDS transmission and prevention among men and women in Uganda.

Method: We analysed data from a nationally-representative population-based cross-sectional survey, the 2016 Uganda demographic and health survey (UDHS), which included all the consenting women aged 15-49 years who were present in sampled households the night before the survey with a sample size of 18,506 women and 5,336 men.

Results: Around 99\% of respondents had heard about HIV/AIDS but only a proportion of $58 \%$ and $54 \%$ of women and men had high knowledge regarding transmission and prevention of HIV/AIDS. After adjusting for socio-demographic factors, women and men in the age group 25-29 years were more likely to have high knowledge regarding transmission and prevention of HIV/AIDS as compared to women and men respectively in 15-19 years ( COR: 1.849; 95\% CI: 1.681-2.033, AOR: 1.489; 95\% CI: 1.323-1.676, (COR: 1.417; 95\% CI: 1.180-1.702).

Conclusion: Continuous supply of antiretroviral therapy (ART) and male circumcision as best ways of reducing HIV transmission, further more health promotion with community involvement can yield a success story.

Keywords: HIV/AIDS; Uganda; Condom use; health education; male circumcision.

(c)2019 The Authors. Published by the JScholar under the terms of the Creative Commons Attribution License http://creativecommons.org/licenses/ by/3.0/, which permits unrestricted use, provided the original author and source are credited. 


\section{Introduction}

According to global HIV statistics 2017, 36.9 million people were living with HIV globally including 1.8 million children. Around $25 \%$ of these same people do not know that they have the virus [1] Since the start of the epidemic, an estimated 77.3 million people have become infected with HIV and 35.4 million people have died of AIDS-related illnesses and approximately 25.7 million people were living with HIV in sub-Saharan Africa by 2017 which is almost 66\% [1] According to Uganda AIDS indicator survey of 2016, around 1.2 million people are living with HIV in Uganda with a prevalence of $6 \%$ of which women are having highest prevalence $7.5 \%$ compared to men $4.3 \%$. Prevention of HIV-1 infection with Early Antiretroviral Therapy (ART), HIV pre and post exposure prophylaxis (PrEP) (PEP), condom use has been a successful story in sub-saharan Africa and throughout the continent. Antiretroviral therapy that reduces viral replication could limit the transmission of human immunodeficiency virus type 1 (HIV-1) in serodiscordant couples [2-7]. Many studies has shown that sexuality education is key in prevention of HIV and related sexually transmitted diseases especially if given to adolescents, schools and many people those that receive sex education are likely not to indulge in risky sexual behaviours $[8,9]$. HIV testing and counselling is so important because it helps to identify those having HIV making it easier for treatment especially home based HIV counselling and testing and also prevent mother-to-child transmission (MTCT) this should also be coupled with counselling about family planning methods other than condom use alone [10-13] and which lowers transmission to others however some studies found that people are scared to find out their status, shy, fear what people might say about them in case they are positive and above all fear of death [14]. There is much evidence that a higher level of education is correlated with having more knowledge about HIV risk when compared with populations of lower education and in such categories transmission of HIV is lower in highly educated as compared to the counterparts [15]. In addition to those peer-education-based interventions, social gatherings, trainings appear to be particularly effective in facilitating the uptake of HIV-related knowledge, particularly pertaining to transmission routes [1617]. However, some studies have found that despite the knowledge levels about HIV seem high, misconception about routes of transmission, HIV/AIDS cure and condom use is still very common [18-19]. Some other studies conducted in Uganda suggested continuous supply of ART and male circumcision as best ways for HIV prevention coupled with community involvement [20-23]. However studies have shown that male circumcision is only effective when the male partner is sero negative so even when the male partner is circumcised when is already positive, this does not reduce transmission of HIV [24]. Another study done in Kenya suggested that exclusive breastfeeding (EBF) (breast milk feeding without additional food or drink, except medicine is likely to reduce the risk of postnatal transmission of HIV from mother to child [25]. It is also much important to create HIV awareness and safe sexual behaviours among female sex workers as one way to prevent the transmission of HIV and this would increase their knowledge about HIV like consistent use of condoms with regular clients and non paying partners hence increasing safe sexual practices aimed at curbing transmission of HIV/AIDS [26]. Other studies have revealed that partnership dissolution in HIV status aware serodiscordant couples reduce the spread of HIV and this is majorly achieved through couple HIV testing and counselling [27, 28]. However, interactions among poverty, low knowledge gender, and health systems affect women's participation in services to prevent HIV transmission from mother to child (PMTCT) also challenges faced by outreach workers for PMTCT in addition stigma, financial costs of travelling to ART centres, non-affordability of infant formula, disclosure, ART use, service design and quality, spouse/partner influence, lack of awareness of the baby's needs, decision-making autonomy in turn affects women's participation in PMTCT services [29-30]. Also some studies have suggested that specific interventions are needed to improve the utilisation of HIV testing amongst heterosexual drug users, particularly amongst non-injection drug users since they are at very high risk of HIV transmission [31]. A study carried out in Congo about male students' perspectives on condom use and concurrent sexual partnerships revealed low knowledge about prevention of sexually transmitted infection since majority of students said that they prefer flesh to flesh sex than using condom and this seems prevalent among school students [32].

\section{Method}

We analysed data from a nationally-representative population-based cross-sectional survey, the 2016 Uganda demographic and health survey (UDHS), which included all the consenting women aged 15-49 years who were present in sampled households the night before the survey. The detailed methods are described elsewhere in UBOS \& ICF international 2016.

\section{Sampling}

The sampling frame used for the 2016 UDHS is the frame of the Uganda National Population and Housing Census 
(NPHC), conducted in 2014; the sampling frame was provided by the Uganda Bureau of Statistics. The census frame is a complete list of all census enumeration areas (EAs) created for the 2014 NPHC. The 2016 UDHS sample was stratified and selected in two stages (NPHC). In the first stage, 697 EAs were selected from the 2014 Uganda NPHC: 162 EAs in urban areas and 535 in rural areas. One cluster from Acholi sub region was eliminated because of land disputes. Households constituted the second stage of sampling.

\section{Variables and measurement}

\section{Independent variables}

Age (15-19, 20-24, 25-29, 30-34, 35-39, 40-44, 45-49),

Education (no education, primary, secondary, higher)

Residence (rural, urban)

Ethnicity ( baganda, banyankore, bakiga, basoga, iteso, lango, others)

Wealth index (poorest, poorer, middle, richer, richest)

Religion (Anglican, catholic, Muslim, seventh day Adventist, Pentecostal/born again, others)

Occupation (not working, skilled work, agric/house hold domestic work, unskilled work)

Media exposure (never, frequently, sometimes)

Marital status (never in union, married, living with partner, widowed, divorced, separated) according to DHS 2016.

\section{Dependent variable}

\section{Knowledge of HIV/AIDS}

\section{Measurement of knowledge}

Study measured knowledge regarding transmission and prevention of HIV/AIDS using questions 1-15 below which were positively and negatively framed.1) Ever heard of AIDS 2) Reduce risk of getting HIV: always use condoms during sex 2) Reduce risk of getting HIV: have 1 sex partner only, who has no other partners 3) Can get HIV from mosquito bites 4) Can get HIV by sharing food with person who has AIDS 5) A healthy looking person can have HIV 6) Condom used during last sex with most recent partner 7) Condom used during last sex with 2nd to most recent partner 8) Condom used during last sex with 3rd to most recent partner 9) Can HIV be transmitted during pregnancy 10) Can HIV be transmitted during pregnancy 11) Can HIV be transmitted during delivery 12) Can HIV be transmitted by breastfeeding 13) Would be ashamed if someone in the family had HIV 14) Can get HIV by witchcraft or supernatural means 15) Drugs to avoid HIV transmission to baby during pregnancy

With these questions we created a composite index of knowledge and this was categorized as high and low knowledge in a dichotomous variable. We adopted the scoring procedure from previous published study of shone [33].That is, a score of 1 was assigned for correct and 0 for incorrect answers (including refused or don't know) and means were categorized as low knowledge $\leq 7.66$ and high knowledge as $>7.66$ for men while for women means were categorized as low knowledge $\leq 8.4$ and high knowledge as $>8.4$ this was translated as low knowledge for scores $\leq 50 \%$ and high knowledge for scores $\geq 51 \%$.

\section{Statistical analysis}

Descriptive and bivariate analysis were conducted to determine association between socio-demographic variables and knowledge of transmission and prevention of HIV/AIDS, we employed survey -weighted logistic regression. For bivariate analysis, we used a Pearson chi-square test to assess significance. Multi-variate adjusted model included with all variables. Any p-value $<0.05$ was considered significant. All statistical analyses were performed using SPSS version 20.

\section{Results}

\section{Descriptive statistics}

Table 1 shows the background characteristics of the respondents of a total of 18,506 women and 5,336 men Majority of respondents were in the age group 15-19 years with $23.0 \%$ followed by $20-24$ years with $20.7 \%$ and the least was $45-49$ years with $6.5 \%$. 39.2\% of the respondents were Catholics, $31 \%$ were Anglicans and Muslims were 12.9\%, 13.3 were born again, 1.6 seventh day Adventists and rest were 1.3\%. Majority of the respondents completed only primary $57.4 \%$ followed by secondary $25.1 \%$ and those who completed higher education were $7.9 \%$ and those completely with no education 9.6\%. Majority of the respondents were Baganda $16.8 \%$ followed by Banyankore and Bakiga while lango only 5.7\% participated and others $44.8 \% .73 .3$ $\%$ were residing in rural and only $26.7 \%$ in urban. Around $45 \%$ of the respondents were involved in agriculture / house hold domestic work while $22.8 \%$ were not involved in any form of work 
Table 1. Background characteristics of the respondents

\begin{tabular}{|c|c|c|c|c|}
\hline Variable & $\%$ & $\mathbf{N}$ & $\%$ & $\mathbf{N}$ \\
\hline \multicolumn{5}{|l|}{ Age } \\
\hline $15-19$ & 23.0 & 4276 & 24.1 & 1270 \\
\hline $20-24$ & 20.7 & 3782 & 17.8 & 944 \\
\hline $25-29$ & 16.5 & 3014 & 13.9 & 740 \\
\hline $30-34$ & 13.7 & 4276 & 13.8 & 737 \\
\hline $35-39$ & 10.9 & 3784 & 9.2 & 497 \\
\hline $40-44$ & 8.7 & 3014 & 9.6 & 492 \\
\hline $45-49$ & 6.5 & 2600 & 6.0 & 363 \\
\hline \multicolumn{5}{|l|}{ Religion } \\
\hline Anglican & 31.2 & 5799 & 33.7 & 1831 \\
\hline Catholic & 39.6 & 7552 & 40.4 & 2201 \\
\hline Muslim & 12.9 & 2166 & 13.5 & 644 \\
\hline Seventh Day Adventist & 1.6 & 292 & 1.4 & 68 \\
\hline Pentecost/ Born again & 13.3 & 2436 & 9.4 & 497 \\
\hline Others & 1.3 & 2697 & 1.6 & 95 \\
\hline \multicolumn{5}{|l|}{ Education } \\
\hline No Education & 9.6 & 2071 & 4.2 & 231 \\
\hline Primary & 57.4 & 10893 & 55.3 & 3047 \\
\hline Secondary & 25.1 & 4213 & 28.0 & 1449 \\
\hline More than a secondary & 7.9 & 1329 & 12.5 & 609 \\
\hline \multicolumn{5}{|l|}{ Ethnicity } \\
\hline Baganda & 16.8 & 2436 & 17.9 & 729 \\
\hline Banyankore & 10.8 & 1687 & 10.9 & 479 \\
\hline Bakiga & 6.8 & 1419 & 6.9 & 398 \\
\hline Basoga & 7.6 & 1161 & 7.5 & 356 \\
\hline Iteso & 7.5 & 1637 & 7.6 & 468 \\
\hline Lango & 5.7 & 1298 & 6.5 & 431 \\
\hline Others & 44.8 & 8868 & 42.7 & 2475 \\
\hline \multicolumn{5}{|l|}{ Residence } \\
\hline Urban & 26.7 & 4379 & 24.9 & 1150 \\
\hline Rural & 73.3 & 14127 & 75.1 & 4186 \\
\hline \multicolumn{5}{|l|}{ Occupation } \\
\hline Not Working & 22.8 & 4077 & 5.8 & 298 \\
\hline Skilled work & 17.2 & 2968 & 15.8 & 704 \\
\hline Agriculture/ hse hold domestic work & 45.4 & 8489 & 48.0 & 2416 \\
\hline Unskilled work & 14.5 & 2936 & 30.4 & 1375 \\
\hline \multicolumn{5}{|l|}{ Wealth Index } \\
\hline Poorest & 17.5 & 3884 & 16.9 & 1057 \\
\hline Poorer & 18.4 & 3640 & 17.7 & 1047 \\
\hline Middle & 18.7 & 3485 & 19.6 & 1049 \\
\hline Richer & 19.9 & 3454 & 22.0 & 1083 \\
\hline Richest & 25.5 & 4043 & 23.7 & 1100 \\
\hline \multicolumn{5}{|l|}{ Marital Status } \\
\hline Never in union & 25.8 & 4738 & 39.0 & 2029 \\
\hline Married & 30.3 & 5813 & 36.1 & 2052 \\
\hline Living with partner & 30.3 & 5566 & 19.3 & 960 \\
\hline Widowed & 2.8 & 523 & .4 & 22 \\
\hline Divorced & 0.8 & 139 & 0.6 & 34 \\
\hline Separated & 9.9 & 1727 & 4.6 & 239 \\
\hline
\end{tabular}


while $17.2 \%$ skilled work and $14.5 \%$ are involved in unskilled work. Approximately $25 \%$ of the respondents were richest while $17.5 \%$ were poorest rest of the respondents lie in the middle of these two categories. Around $30.3 \%$ of respondents were either married or living with partner while $25.8 \%$ were never in union and $2.8 \%$ and $0.8 \%$ were widowed and divorced respectively and 9.9 separated.

Table 2 shows distribution of HIV/AIDS knowledge among women in relation to socio-demographic factors. There was a strong association between age and knowledge regarding transmission and prevention of HIV/AIDS with $\mathrm{p}=0.00$. It was shown that in the age group of 25-29 years $63.3 \%$ have high knowledge while $15-19$ years $48.3 \%$ have low knowledge also those between 45-49 years $58 \%$ had low knowledge. Religion was associated with knowledge regarding transmission and prevention of HIV/AIDS with $\mathrm{p}=0.01$. Around $61.1 \%$ of Muslims were found to have high knowledge while seventh day Adventists had the lowest knowledge. There was association between education and knowledge regarding transmission and prevention of HIV/AIDS with $\mathrm{p}=0.01$. It was seen that $60.0 \%$ of respondents with secondary education had high knowledge as compared to primary and higher education while $44.8 \%$ of respondents with no education had low knowledge. There was a strong association between residence, wealth index, marital status, occupation, ethnicity, and media exposure with $\mathrm{p}<0.05$ and knowledge regarding transmission and prevention of HIV/AIDS.

Table 3 shows distribution of HIV/AIDS knowledge among men in relation to socio-demographic factors. Around $60.1 \%$ of men had high knowledge in age group 25-29 years and was strongly associated with knowledge regarding transmission and prevention of HIV/AIDS. There was no any association between religion, education, residence and knowledge regarding transmission and prevention of HIV/AIDS ( $\mathrm{P}=0.55,0.31,0.91)$ respectively. Association was also seen in wealth index, marital status, occupation, ethnicity and media exposure.

Table 4 shows Unadjusted and adjusted odds ratios for factors associated with knowledge regarding transmission and prevention of HIV/AIDS among women with socio-demographic factors. Age was statistically significant and women in the age group 25-29 years were more likely to have more knowledge regarding transmission and prevention of HIV/AIDS as compared to women in 15-19 years ( OR: 1.85 ; 95\% CI: 1.68 2.03, aOR: 1.49; 95\% CI: 1.32-1.68). Being Pentecost/born again were less likely to have knowledge regarding transmission and prevention of HIV/AIDS as compared to Anglican aOR: 0.90:
95\% CI: 0.82-0.99. Ethnicity was statistically significant and Banyankore were 0.69 times less likely to have knowledge regarding transmission and prevention of HIV/AIDS (aOR: 0.69; 95\% CI: 0.62-0.79) while Iteso was 0.46 times less likely to have knowledge regarding transmission and prevention of HIV/AIDS (OR: 0. 46; 95\% CI: 0.40-0.52). According to education those having primary were 1.12 times more likely to have knowledge compared to those with no education (aOR: 1.12; 95\% CI: 1.06-1.32). According to wealth index, those who are in middle, richer and richest were $1.23,1.37$ and 1.38 times more likely than poorest to have more knowledge regarding transmission and prevention of HIV/AIDS. Those who are widowed are more likely to have high knowledge regarding transmission and prevention of HIV/ AIDS (OR: 1.79; 95\% CI: 1.48-2.16) after adjusting ( aOR: 1.59; 95\% CI: 1.29-1.96). According to occupation, those with skilled work and agriculture were 1.37 and 1.20 respectively more likely to have knowledge regarding transmission and prevention of HIV/AIDS. (OR: 1.37; 95\% CI: 1.25-1.51), (OR: 1.20 95\% CI: 1.12-1.29) respectively. Media exposure was statistically significant with those frequently exposed to media were more likely to have more knowledge compared to those never exposed to media (OR: 0.82; 95\% CI: 0.76-0.88).

Table 5 shows unadjusted and adjusted odds ratios for factors associated with knowledge regarding transmission and prevention of HIV/AIDS among men Age group 25-29 years was statistically significant and they were likely to have more knowledge as compared to the reference category (COR: 1.42; 95\% CI: 1.18-1.70) while those in age group 45-49 years were less likely to have knowledge (aOR: 0.69; 95\% CI: 0.50-0.97). According to wealth index, those who are in the middle and richest were more likely to have knowledge regarding transmission and prevention of HIV/ AIDS (COR: 1.32; 95\% CI: 1.09-1.57) and (COR: 1.21; 95\% CI: 1.02-1.43) respectively. According to occupation, those with unskilled work were more likely to have knowledge (COR: 1.59; 95\% CI: 1.24-2.06). 
Table 2 percentage distribution of kno wledge by background characteristics among women

\begin{tabular}{|c|c|c|c|c|}
\hline Variables & Low & High & Total & P-Value \\
\hline & $\%$ & $\%$ & $\mathrm{~N}$ & \\
\hline \multicolumn{5}{|l|}{ Age } \\
\hline $15-19$ & 51.7 & 48.3 & 4276 & \multirow{7}{*}{0.00} \\
\hline $20-24$ & 39.9 & 60.1 & 3782 & \\
\hline $25-29$ & 36.7 & 63.3 & 3014 & \\
\hline $30-34$ & 37.1 & 62.9 & 2600 & \\
\hline $35-39$ & 39.4 & 60.6 & 2029 & \\
\hline $40-44$ & 39 & 61 & 1621 & \\
\hline $45-49$ & 41.7 & 58.3 & 1184 & \\
\hline \multicolumn{5}{|l|}{ Religion } \\
\hline Anglican & 41 & 59 & 5799 & \multirow{6}{*}{0.01} \\
\hline Catholic & 42.4 & 57.6 & 7552 & \\
\hline Muslim & 38.9 & 61.1 & 2166 & \\
\hline Seventh Day Adventist & 43.8 & 56.2 & 292 & \\
\hline Pentecostal/ born again & 43.6 & 56.4 & 2436 & \\
\hline Others & 41.3 & 58.7 & 261 & \\
\hline \multicolumn{5}{|l|}{ Education } \\
\hline No Education & 44.8 & 55.2 & 2071 & \multirow{4}{*}{0.01} \\
\hline Primary & 41.8 & 58.2 & 10893 & \\
\hline Secondary & 40 & 60.0 & 4213 & \\
\hline higher & 42.6 & 57.4 & 1329 & \\
\hline \multicolumn{5}{|l|}{ Residence } \\
\hline Urban & 38.9 & 61.1 & 4379 & \multirow[b]{2}{*}{0.00} \\
\hline Rural & 42.7 & 57.3 & 14127 & \\
\hline \multicolumn{5}{|l|}{ Wealth Index } \\
\hline Poorest & 49.1 & 50.9 & 3884 & \multirow{5}{*}{0.00} \\
\hline Poorer & 43.8 & 56.2 & 3640 & \\
\hline Middle & 40.2 & 59.8 & 3485 & \\
\hline Richer & 38.7 & 61.3 & 3454 & \\
\hline Richest & 38.5 & 61.5 & 4043 & \\
\hline \multicolumn{5}{|l|}{ Marital Status } \\
\hline Never in union & 50.7 & 49.3 & 4738 & \multirow{6}{*}{0.00} \\
\hline Married & 42.3 & 57.7 & 5813 & \\
\hline Living with partner & 36 & 64 & 5566 & \\
\hline Widowed & 36.5 & 63.5 & 523 & \\
\hline Divorced & 42.8 & 57.2 & 139 & \\
\hline No longer living together/separated & 35.1 & 64.9 & 1727 & \\
\hline \multicolumn{5}{|l|}{ Occupation } \\
\hline Not working & 45.7 & 54.3 & 4077 & \multirow{4}{*}{0.00} \\
\hline Skilled Work & 38.0 & 62.0 & 2968 & \\
\hline Agriculture/Hse Hold Domestic Work & 41.1 & 58.9 & 8489 & \\
\hline Unskilled Work & 41.4 & 58.6 & 2936 & \\
\hline
\end{tabular}




\begin{tabular}{|c|c|c|c|c|}
\hline Variables & Low & High & Total & P-Value \\
\hline & $\%$ & $\%$ & $\mathrm{~N}$ & \\
\hline \multicolumn{5}{|l|}{ Ethnicity } \\
\hline Baganda & 33.5 & 66.5 & 2436 & \\
\hline Banyankore & 42.6 & 57.4 & 1687 & \\
\hline Bakiga & 34.3 & 65.7 & 1419 & \\
\hline Basoga & 36.3 & 63.7 & 1161 & 0.00 \\
\hline Iteso & 52.5 & 47.5 & 1637 & \\
\hline Lango & 48.1 & 51.9 & 1298 & \\
\hline Others & 43.9 & 56.1 & 8868 & \\
\hline \multicolumn{5}{|c|}{ Media Exposure } \\
\hline Never & 45.7 & 54.3 & 4188 & \\
\hline Frequently & 39.9 & 60.1 & 794 & 0.00 \\
\hline Some times & 40.7 & 59.3 & 13524 & \\
\hline TOTAL & 41.7 & 58.3 & 18506 & \\
\hline
\end{tabular}

Table 3 percentage distribution of knowledge by background characteristics among men

\begin{tabular}{|c|c|c|c|c|}
\hline Variables & Low & High & Total & P-Value \\
\hline & $\%$ & $\%$ & $\mathrm{~N}$ & \\
\hline \multicolumn{5}{|l|}{ Age } \\
\hline $15-19$ & 48.5 & 51.5 & 1270 & \multirow{8}{*}{0.00} \\
\hline $20-24$ & 41.8 & 58.2 & 944 & \\
\hline $25-29$ & 39.9 & 60.1 & 740 & \\
\hline $30-34$ & 41.1 & 58.9 & 737 & \\
\hline $35-39$ & 49.2 & 50.8 & 497 & \\
\hline $40-44$ & 43.2 & 56.2 & 492 & \\
\hline $45-49$ & 52.9 & 47.1 & 363 & \\
\hline $50-54$ & 53.5 & 46.5 & 293 & \\
\hline \multicolumn{5}{|l|}{ Religion } \\
\hline Anglican & 45.1 & 54.9 & 1831 & \multirow{6}{*}{0.55} \\
\hline Catholic & 44.0 & 56.0 & 2201 & \\
\hline Muslim & 47.0 & 53.0 & 644 & \\
\hline Seventh Day Adventist & 47.1 & 52.9 & 68 & \\
\hline Pentecostal/ born again & 48.0 & 52.0 & 497 & \\
\hline Others & 43.5 & 56.5 & 95 & \\
\hline \multicolumn{5}{|l|}{ Education } \\
\hline No Education & 46.4 & 53.6 & 231 & \multirow{4}{*}{0.31} \\
\hline Primary & 45.2 & 54.3 & 3047 & \\
\hline Secondary & 43.7 & 56.3 & 1449 & \\
\hline higher & 48 & 52 & 609 & \\
\hline \multicolumn{5}{|l|}{ Residence } \\
\hline Urban & 45.1 & 54.9 & 1150 & \multirow{3}{*}{0.91} \\
\hline Rural & 45.2 & 54.8 & 4186 & \\
\hline \multicolumn{4}{|l|}{ Wealth Index } & \\
\hline Poorest & 49.2 & 50.8 & 1057 & \\
\hline
\end{tabular}




\begin{tabular}{|c|c|c|c|c|}
\hline Variables & Low & High & Total & P-Value \\
\hline & $\%$ & $\%$ & $\mathrm{~N}$ & \\
\hline Poorer & 45.5 & 54.5 & 1047 & \\
\hline Middle & 45.4 & 57.6 & 1049 & \\
\hline Richer & 45.2 & 54.8 & 1083 & 0.05 \\
\hline Richest & 44.5 & 55.5 & 1100 & \\
\hline \multicolumn{5}{|l|}{ Marital Status } \\
\hline Never in union & 46.9 & 53.1 & 2029 & \multirow{6}{*}{0.00} \\
\hline Married & 48.3 & 51.7 & 2052 & \\
\hline Living with partner & 37.6 & 62.4 & 960 & \\
\hline Widowed & 30.2 & 69.8 & 22 & \\
\hline Divorced & 51.0 & 49.0 & 34 & \\
\hline $\begin{array}{l}\text { No longer living togeth- } \\
\text { er/separated } \\
\text { Occupation }\end{array}$ & 38.9 & 61.1 & 239 & \\
\hline Not working & 52.6 & 47.4 & 298 & \multirow{4}{*}{0.00} \\
\hline Skilled Work & 48.2 & 51.8 & 704 & \\
\hline hold domestic Work & 46.7 & 53.3 & 2416 & \\
\hline Unskilled Work & 41.0 & 59.0 & 1375 & \\
\hline \multicolumn{5}{|l|}{ Ethnicity } \\
\hline Baganda & 41.7 & 58.3 & 729 & \multirow{7}{*}{0.00} \\
\hline Banyankore & 39.5 & 60.5 & 479 & \\
\hline Bakiga & 38.9 & 61.1 & 398 & \\
\hline Basoga & 43.2 & 56.8 & 356 & \\
\hline Iteso & 61.0 & 39.0 & 468 & \\
\hline Lango & 42.9 & 57.1 & 431 & \\
\hline Others & 47.0 & 53.0 & 2475 & \\
\hline \multicolumn{5}{|l|}{ Media Exposure } \\
\hline Never & 47.7 & 52.3 & 525 & \multirow{3}{*}{0.01} \\
\hline Frequently & 44.2 & 55.8 & 4294 & \\
\hline Some times & 51.4 & 48.6 & 517 & \\
\hline Total & 56 & 54 & 5336 & \\
\hline
\end{tabular}


Table 4. Unadjusted and adjusted odds ratios for factors associated with knowledge regarding transmission and prevention of HIV/AIDS among women.

\begin{tabular}{|c|c|c|}
\hline Variables & $\begin{array}{l}\text { Crude ( Un adjusted odds) } \\
(95 \% \text { C.I })\end{array}$ & Adjusted odds ratio (95\% C.I) \\
\hline \multicolumn{3}{|c|}{ 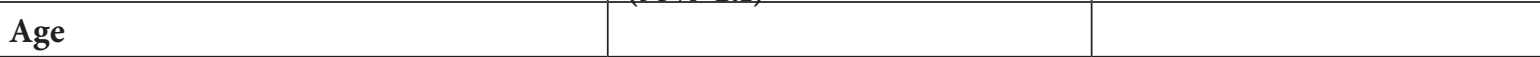 } \\
\hline \multicolumn{3}{|l|}{ 15-19 Ref } \\
\hline $20-24$ & $1.62(1.48-1.77)^{\star * *}$ & $1.36(1.23-1.51)^{\star * *}$ \\
\hline $25-29$ & $1.85(1.68-2.03)^{* * *}$ & $1.49(1.32-1.68)^{* * *}$ \\
\hline $30-34$ & $1.82(1.65-2.01)^{\star * *}$ & $1.47(1.29-1.67)^{\star * *}$ \\
\hline $35-39$ & $1.65(1.48-1.84)^{* * *}$ & $1.32(1.15-1.51)^{\star * *}$ \\
\hline $40-44$ & $1.67(1.49-1.88)^{\star * *}$ & $1.35(1.17-1.56)^{\star * *}$ \\
\hline $45-49$ & $1.51(1.33-1.72)^{\star * \star}$ & $1.22(1.04-1.43)^{\star \star}$ \\
\hline \multicolumn{3}{|l|}{ Residence } \\
\hline \multicolumn{3}{|l|}{ Urban Ref } \\
\hline Rural & $0.85(0.79-0.91)$ & $0.96(0.88-1.04)$ \\
\hline \multicolumn{3}{|l|}{ Religion } \\
\hline \multicolumn{3}{|l|}{ Anglican Ref } \\
\hline Catholic & $0.94(0.88-1.01)$ & $0.97(0.91-1.05)$ \\
\hline Muslim & $1.09(0.99-1.20)$ & $0.97(0.87-1.07)$ \\
\hline Seventh Day Adventist & $0.89(0.70-1.12)$ & $0.82(0.64-1.04)$ \\
\hline Pentecost/ born again & $0.89(0.81-0.98)^{*}$ & $0.90(0.81-0.99)^{*}$ \\
\hline Others & $0.98(0.75-1.28)$ & $1.02(0.78-1.34)$ \\
\hline \multicolumn{3}{|l|}{ Ethnicity } \\
\hline \multicolumn{3}{|l|}{ Baganda Ref } \\
\hline Banyankore & $0.67(0.60-0.76)^{* * *}$ & $0.69(0.61-0.78)^{* * *}$ \\
\hline Bakiga & $0.96(0.84-1.10)$ & $1.03(0.89-1.19)$ \\
\hline Basoga & $0.88(0.77-1.01)$ & $0.96(0.84-1.10)$ \\
\hline Iteso & $0.45(0.40-0.51)^{\star * *}$ & $0.55(0.47-0.63)^{\star * *}$ \\
\hline Lango & $0.54(0.47-0.63)^{\star * *}$ & $0.66(0.57-0.77)^{\star * *}$ \\
\hline Others & $0.64(0.59-0.70)^{\star * *}$ & $0.72(0.65-0.79)^{\star * *}$ \\
\hline \multicolumn{3}{|l|}{ Education } \\
\hline \multicolumn{3}{|l|}{ No Education Ref } \\
\hline Primary & $0.92(0.79-1.06)$ & $1.18(1.06-1.32)^{*}$ \\
\hline Secondary & $1.04(0.93-1.16)$ & $1.13(0.99-1.29)$ \\
\hline higher & $1.11(0.99-1.26)$ & $0.89(0.75-1.05)$ \\
\hline \multicolumn{3}{|l|}{ Wealth Index } \\
\hline \multicolumn{3}{|l|}{ Poorest Ref } \\
\hline Poorer & $1.24(1.12-1.36)^{\star * *}$ & $1.17(1.06-1.29)^{\star}$ \\
\hline Middle & $1.43(1.30-1.58)^{\star * *}$ & $1.29(1.17-1.44)^{\star * *}$ \\
\hline Richer & $1.53(1.39-1.68)^{* * *}$ & $1.37(1.23-1.53)^{\star * *}$ \\
\hline Richest & $1.54(1.41-1.69)^{\star * *}$ & $1.38(1.22-1.56)^{\star * *}$ \\
\hline \multicolumn{3}{|l|}{ Marital Status } \\
\hline \multicolumn{3}{|l|}{ Never in union Ref } \\
\hline Married & $1.40(1.29-1.52)^{\star * *}$ & $1.22(1.09-1.36)^{\star * *}$ \\
\hline Living with partner & $1.83(1.69-1.98)^{\star * *}$ & $1.50(1.36-1.66)^{\star * *}$ \\
\hline
\end{tabular}




\begin{tabular}{|l|l|l|}
\hline Widowed & $1.79(1.49-2.16)^{\star * *}$ & $1.59(1.29-1.96)^{* * *}$ \\
\hline Divorced & $1.38(0.98-1.93)$ & $1.12(0.78-1.59)$ \\
\hline No longer living together & $1.90(1.70-2.13)^{\star * *}$ & $1.55(1.36-1.76)^{* * *}$ \\
\hline Occupation & & \\
\hline Not working Ref & & \\
\hline Skilled Work & $1.37(1.25-1.51)^{\star * *}$ & $1.08(0.98-1.20)$ \\
\hline Agriculture/Hse Hold Domestic Work & $1.20(1.12-1.29)^{\star * *}$ & $1.08(0.99-1.17)$ \\
\hline Unskilled Work & $1.19(1.08-1.31)^{\star *}$ & $1.05(0.95-1.17)$ \\
\hline Media Exposure & & \\
\hline Never Ref & & $1.05(0.89-1.25)$ \\
\hline Frequently & $0.82(0.76-0.88)^{\star * *}$ & $1.07(0.99-1.15)$ \\
\hline Sometimes & $1.03(0.89-1.18)$ & \\
\hline
\end{tabular}

Note: Ref is the reference category, level of significance ${ }^{\star} \mathrm{p}$-value of $<0.05,{ }^{* *} \mathrm{p}$-value of $<0.01,{ }^{* * *} \mathrm{p}$-value of $<0.001$

Table 5. Unadjusted and adjusted odds ratios for factors associated with knowledge regarding transmission and prevention of HIV/AIDS among men.

\begin{tabular}{|c|c|c|}
\hline Variables & $\begin{array}{l}\text { Crude(Unadjusted odds ratio) } \\
(95 \% \text { C.I) }\end{array}$ & Adjusted odds ratio (95\% C.I) \\
\hline \multicolumn{3}{|l|}{ Age } \\
\hline \multicolumn{3}{|l|}{ 15-19 Ref } \\
\hline $20-24$ & $1.31(1.11-1.55)^{*}$ & $1.23(1.00-1.49)^{*}$ \\
\hline $25-29$ & $1.42(1.18-1.70) * * *$ & $1.22(0.94-1.57)$ \\
\hline $30-34$ & $1.35(1.12-1.62)^{* *}$ & $1.15(0.87-1.51)$ \\
\hline $35-39$ & $0.97(0.79-1.19)$ & $0.82(0.61-1.11)$ \\
\hline $40-44$ & $1.24(1.01-1.52)^{*}$ & $1.09(0.81-1.48)$ \\
\hline $45-49$ & $0.84(0.66-1.07)$ & $0.69(0.50-0.97)^{*}$ \\
\hline \multicolumn{3}{|l|}{ Ethnicity } \\
\hline \multicolumn{3}{|l|}{ Baganda Ref } \\
\hline Banyankore & $1.09(0.89-1.35)$ & $1.15(0.90-1.46)$ \\
\hline Bakiga & $1.12(0.88-1.44)$ & $1.13(0.87-1.48)$ \\
\hline Basoga & $0.94(0.74-1.19)$ & $0.99(0.76-1.27)$ \\
\hline Iteso & $0.45(0.36-0.58) * * *$ & $0.49(0.38-0.65)^{* * *}$ \\
\hline Lango & $0.95(0.74-1.22)$ & $1.06(0.79-1.40)$ \\
\hline Others & $0.81(0.69-0.94)$ & $0.87(0.72-1.04)$ \\
\hline \multicolumn{3}{|c|}{ Wealth Index } \\
\hline \multicolumn{3}{|l|}{ Poorest Ref } \\
\hline Poorer & $1.16(0.96-1.39)$ & $1.02(0.83-1.24)$ \\
\hline Middle & $1.32(1.09-1.57)^{*}$ & $1.15(0.98-1.41)$ \\
\hline Richer & $1.17(0.98-1.39)$ & $0.99(0.81-1.23)$ \\
\hline Richest & $1.21(1.02-1.43)^{*}$ & $1.14(0.89-1.46)$ \\
\hline \multicolumn{3}{|c|}{ Marital Status } \\
\hline \multicolumn{3}{|c|}{ Never in union Ref } \\
\hline Married & $0.95(0.83-1.07)$ & $1.09(0.88-1.36)$ \\
\hline
\end{tabular}




\begin{tabular}{|l|l|l|}
\hline Living with partner & $1.46(1.26-1.70)^{* * *}$ & $1.44(1.15-1.79)^{*}$ \\
\hline Widowed & $2.04(0.77-5.39)$ & $2.83(1.02-7.84)^{*}$ \\
\hline Divorced & $0.85(0.43-1.67)$ & $0.99(0.48-2.07)$ \\
\hline No longer living together/separated & $1.39(1.06-1.82)^{* *}$ & $1.47(1.05-2.06)^{*}$ \\
\hline Occupation & & \\
\hline Not working Ref & & $1.05(0.77-1.42)$ \\
\hline Skilled Work & $1.19(0.90-1.56)$ & $1.19(0.92-1.56)$ \\
\hline $\begin{array}{l}\text { Agriculture/House Hold Domestic } \\
\text { Work }\end{array}$ & $1.26(0.99-1.62)$ & $1.34(1.02-1.76)^{*}$ \\
\hline Unskilled Work & $1.59(1.24-2.06)^{* * *}$ & \\
\hline Media Exposure & & $1.13(0.83-1.54)$ \\
\hline Never Ref & & $1.19(0.87-1.66)$ \\
\hline Frequently & $1.15(0.95-1.39)$ & \\
\hline Sometimes & $0.86(0.67-1.11)$ & \\
\hline
\end{tabular}

Note: Ref is the reference category, level of significance ${ }^{\star} p$-value of $<0.05$, ${ }^{* *} p$-value of $<0.01,{ }^{* *} p$-value of $<0.001$

\section{Discussion}

Our study examined the factors associated with knowledge regarding transmission and prevention of HIV/AIDS in Uganda. The present study examined both men and women knowledge. The results show that there is improved HIV knowledge and similar study by Shona and kadengye [33] support this finding. The study from the national population based survey found out that over $99 \%$ of both women and men ever heard of AIDS but only $58 \%$ and $54 \%$ had high knowledge about transmission and prevention of HIV, this means health education need to be scaled up to achieve 90-90-90 of UNAIDS 2020 target. According to kadengye and shone [33] 40\% of the women had high knowledge which means there is slight increase in the knowledge regarding transmission and discrimination. These results were consistent with pelterzer [14].

Previous studies have showed that education play a big role in curbing HIV/AIDS and this is consistent with this study as we found education statistically associated with HIV knowledge $[14,34]$.Many studies showed that Early Antiretroviral Therapy (ART) and condom use can prevent transmission of HIV/ AIDS and this can lower the incidence of the infection hence controlling the spread of the disease [2-5]. Previous studies revealed that testing and counselling have shown a successful story since this helps in early case detection and early treatment which has played a major role in preventing mother to child transmission (MTCT) therefore this should continue to be scaled up as a public health strategy to prevent transmission of HIV/AIDS [10], [11]. However, some studies have found that despite the knowledge levels about HIV seem improving, misconception about routes of transmission, HIV/AIDS cure and condom use is still very common in our study we found that $24 \%$ of respondents who still believe HIV can be transmitted by mosquito bites [18-19]. A limitation to our analysis is that we analysed knowledge of HIV/AIDS but were not able to assess actual HIV status.

\section{Conclusion}

Women and men who are residing in urban with higher education, richest and having skilled work were less likely to express stigma and discriminatory attitudes towards PLWHA and have high knowledge. Around 58\% and 54\% of women and men respectively had high knowledge about transmission and prevention of HIV/AIDS. Continuous supply of ART and male circumcision as best ways for HIV prevention coupled with community involvement. Scale up voluntary counselling and testing and increasing condom use as one way of preventing the transmission of HIV and health education need to be scaled up to achieve 90-90-90 of UNAIDS 2020 target. Sex education should be included in the school syllabus this will reduce misconceptions surrounding HIV/AIDS transmission and improve early interventions (primary intervention) such as abstinence. Government should not enact laws that criminalise gays, sex workers, injecting drug users because these are high risk groups that are highly responsible for increase of the infection. Criminalising them makes prevention efforts very difficult since they will not show up for treatment. 
Notes

Compliance with ethical standards

Ethical considerations

This article is based on the secondary analysis of data which is available to all on demand for research purposes. Hence no compliance with ethical standards was required but we obtained permission from DHS division at ICF international from were data was downloaded.

\section{References}

1. (2018) U.S depertment of Health \& Human services. Global statistics (Web article).

2. Cohen MS, Chen YQ, McCauley M, Gamble T, Hosseinipour MC, Kumarasamy N \& Godbole SV (2011) Prevention of HIV-1 infection with early antiretroviral therapy. New England journal of medicine 365: 493-505.

3. Attia S, Egger M, Müller M, Zwahlen M, \& Low N (2009) Sexual transmission of HIV according to viral load and antiretroviral therapy: systematic review and meta-analysis. Aids 23: $1397-1404$.

4. Jain A, Tobey E, Ismail H, \& Erulkar A (2018) Condom use at last sex by young men in Ethiopia: the effect of descriptive and injunctive norms. Reproductive Health 15:164

5. Bareki P, \& Tenego T (2018) Assessment of knowledge, attitudes and practices of HIV post exposure prophylaxis among the doctors and nurses in Princess Marina Hospital, Gaborone: a cross-sectional study. Pan African Medical Journal 30: 233.10556 .

6. Kambutse I, Igiraneza G, \& Ogbuagu O (2018) Perceptions of HIV transmission and pre-exposure prophylaxis among health care workers and community members in Rwanda. PLOS ONE 13: e0207650.

7. Chibawara T, Mbuagbaw L, Kitenge M, \& Nyasulu P (2019) Effects of antiretroviral therapy in HIV-positive adults on new HIV infections among young women: a systematic review protocol. Systematic Reviews 8.

8. Haberland N, \& Rogow D (2015). Sexuality education: emerging trends in evidence and practice. Journal of adolescent health 56: S15-S21.

9. Sekirlme W K, Tamale J, Lule JC, \& Wabwire-Mangen F (2001) Knowledge, attitude and practice about sexually transmitted diseases among University students in Kampala 1: 7.

10. Haruna T S, Assenga E, \& Shayo J (2018) A qualitative study on the voluntariness of counselling and testing for HIV amongst antenatal clinic attendees: do women have a choice?

\section{BMC Medical Ethics 19: 92.}

11. Pokharel R, Bhattarai G, Shrestha N, \& Onta S (2018) Knowledge and utilization of family planning methods among people living with HIV in Kathmandu, Nepal. BMC Health Services Research 18: 836.

12. Goggin K, Finocchario-Kessler S, Staggs V, Woldetsadik MA, Wanyenze RK, et al. (2015) Attitudes, Knowledge, and Correlates of Self-Efficacy for the Provision of Safer Conception Counseling Among Ugandan HIV Providers. AIDS Patient Care and STDs 29: 651-660.

13. Dieffenbach C W, \& Fauci AS (2009) Universal voluntary testing and treatment for prevention of HIV transmission Jama 301: 2380-2382.

14. Mohlabane N, Peltzer K, Mwisongo A, Ntsepe Y, Tutshana B, Rooyen HV, \& Knight L (2015) Quality of HIV counselling in South Africa. Journal of Psychology 6: 19-31.

15. Sidhu A, Kakkar R, \& Alenezi O (2019) The Management of Newly Diagnosed HIV in a Sudanese Refugee in Canada: Commentary and Review of Literature. Reviews on Recent Clinical Trials 14: 61-65.

16. Hoang C D, Tran BX, Pham MD, Nguyen LH, Do HN, Vuong QH, et al. (2019) HIV- and AIDS-related knowledge and attitude of residents in border regions of Vietnam. Harm Reduction Journal 16.

17. Nguyen TMT, Tran BX, Fleming M, Pham MD, Nguyen LT, et al. (2019) HIV knowledge and risk behaviors among drug users in three Vietnamese mountainous provinces. Substance Abuse Treatment, Prevention, and Policy 14.

18. Rukundo A, Muwonge MM, Mugisha D, Aturwanaho D, Kasangaki A, et al. (2016) Knowledge, Attitudes and Perceptions of Secondary School Teenagers towards HIV Transmission and Prevention in Rural and Urban Areas of Central Uganda. Health 08: 937-952.

19. Tavoosi A, Zaferani A, Enzevaei A, Tajik P, \& Ahmadinezhad Z (2004) Knowledge and attitude towards HIV/AIDS among Iranian students. BMC Public Health 4.

20. Mukudu H, Dietrich J, Otwombe K, Manentsa M, Hlongwane K, Haas-Kogan M, Martinson N (2019) Voluntary medical male circumcision (VMMC) for prevention of heterosexual transmission of HIV and risk compensation in adult males in Soweto: Findings from a programmatic setting PLOS ONE 14.

21. Mati K, Adegoke KK, \& Salihu H M (2016) Factors associated with married women's support of male circumcision for HIV prevention in Uganda: a population based cross-sectional study. BMC Public Health 16. 
22. Lubega M, Nakyaanjo N, Nansubuga S, Hiire E, Kigozi

G, et al. (2015) Understanding the socio-structural context of high HIV transmission in kasensero fishing community, South Western Uganda. BMC Public Health 15.

23. Chang LW, Mbabali I, Kong X, Hutton H, Amico KR, et al. (2017) Impact of a community health worker HIV treatment and prevention intervention in an HIV hotspot fishing community in Rakai, Uganda (mLAKE): study protocol for a randomized controlled trial. Trials 18.

24. Wawer MJ, Makumbi F, Kigozi G, Serwadda D, Watya S, et al. (2009) Circumcision in HIV-infected men and its effect on HIV transmission to female partners in Rakai, Uganda: a randomised controlled trial. The Lancet 374: 229-237.

25. Okanda J, Otieno G, Kinuthia J, Kohler P. \& John-Stewart G (2018) Higher likelihood of 6-months exclusive breastfeeding among HIV infected than uninfected mothers: a household survey in Kenya. International Breastfeeding Journal 13.

26. Kakchapati S, Gautam N, Kc K, \& Rawal BB (2018) HIV awareness and safe sexual behaviors among female sex workers in Kathmandu valley of Nepal. HIV/AIDS - Research and Palliative Care 10: 157-166.

27. Reniers G, \& Armbruster B (2012) HIV Status Awareness, Partnership Dissolution and HIV Transmission in Generalized Epidemics. PLoS ONE 7.

28. Ramlagan S, Matseke G, Rodriguez VJ, Jones DL, Peltzer K, Ruiter RAC, et al. (2018) Determinants of disclosure and non-disclosure of HIV-positive status, by pregnant women in rural South Africa. SAHARA-J: Journal of Social Aspects of HIV/ AIDS 15:155-163.

29. Yourkavitch J, Hassmiller Lich K, Flax V L, Okello ES, Kadzandira J, Katahoire AR, et al. (2018) Interactions among poverty, gender, and health systems affect women's participation in services to prevent HIV transmission from mother to child: A causal loop analysis. PLOS ONE 13: e0197239.

30. Alemu YM, Habtewold TD, \& Alemu SM (2018) Mother's knowledge on prevention of mother-to-child transmission of HIV, Ethiopia: A cross sectional study. PLOS ONE 13:e0203043.

31. Jiang Z, Xiu C, Yang J, Zhang X, Liu M, Chen X, \& Liu D (2018) HIV test uptake and related factors amongst heterosexual drug users in Shandong province, China. PLOS ONE 13:e0204489.

32. Mulumeoderhwa M (2018) 'It's not good to eat a candy in a wrapper': male students' perspectives on condom use and concurrent sexual partnerships in the eastern Democratic Republic of Congo. SAHARA-J: Journal of Social Aspects of HIV/ AIDS 15: 89-102.
33. Kadengye DT, \& Dalal S (2019) Social intolerance, risky sexual behaviors and their association with HIV knowledge among Ugandan adults: results from a national survey. AIDS care 31: 250-254.

34. Yaya S, Bishwajit G, Danhoundo G, ShahV, \& Ekholuenetale M (2016) Trends and determinants of HIV/AIDS knowledge among women in Bangladesh. BMC Public Health 16: 812 .
Submit your manuscript to a JScholar journal and benefit from:

ब Convenient online submission

- Rigorous peer review

I Immediate publication on acceptance

ब Open access: articles freely available online

I High visibility within the field

q Better discount for your subsequent articles

Submit your manuscript at http://www.jscholaronline.org/submit-manuscript.php 